\title{
AN INTEGRATED MICROELECTROMECHANICAL RESONANT OUTPUT GYROSCOPE
}

\author{
Ashwin A. Seshia ${ }^{*}$, Roger T. Howe ${ }^{*}$ and Stephen Montague ${ }^{\dagger}$ \\ *497 Cory Hall, Dept. of Electrical Engineering and Computer Sciences, University of California, Berkeley, CA 94720, USA. \\ ${ }^{\dagger}$ Sandia National Laboratories, Albuquerque, NM 87185, USA.
}

\begin{abstract}
We describe the principle of operation and experimental characterization of an integrated micromechanical vibratory rate gyroscope based on resonant sensing of the Coriolis force. The new design has several advantages over rate gyroscopes that utilize open-loop displacement sensing for rotation rate measurement. Some of these advantages include simpler dynamics and control, improved scale factor stability, large dynamic range, high resolution, and a quasidigital FM output. A z-axis integrated surface-micromachined gyroscope fabricated at the Sandia National Laboratories has a measured noise floor of $0.3 \mathrm{deg} / \mathrm{sec} / \sqrt{\mathrm{Hz}}$.
\end{abstract}

Keywords: Gyroscope, rate sensor, resonant sensing, surface micromachining.

\section{INTRODUCTION}

A gyroscope is a sensor used to detect angular motion. Several micromechanical gyroscope devices have been reported in literature [1]. Most of these devices operate on the principle of detecting an induced Coriolis acceleration on a proof mass that vibrates along a direction orthogonal to the axis about which the input rotation is applied. A variety of sensing techniques [1] including optical, capacitive, tunneling and piezoresistive have been used to estimate the Coriolis force, and hence the rotation rate, by measuring the displacement of the proof mass in a direction orthogonal to both the driven motion and the axis about which rotational motion is to be sensed. The design of such a device must allow for nearly equal compliance of the proof mass along two orthogonal directions.

Two important parameters that define gyroscope performance are the scale factor (sensitivity to the measurand) and the resolution. In addition, it is required that the sensor output be insensitive to parameters other than the measurand especially ambient environmental parameters such as temperature and pressure.

The scale factor $(S)$ for gyroscopes utilizing open-loop displacement sensing can be expressed as a ratio of the measured displacement $(y)$ in the sensing direction to the rotation rate $\left(\Omega_{z}\right)$ to be sensed [2]:

$$
S=\frac{y}{\Omega_{z}}=\frac{2 X_{o} \omega_{x}}{\left(\omega_{y}^{2}-\omega_{x}^{2}\right)+j \cdot\left(\frac{\omega_{x} \omega_{y}}{Q_{y}}\right)}
$$

Gyroscopes that utilize displacement sensing to determine rotation rate often operate under the condition of matched modes $\left(\omega_{x}=\omega_{y}\right)$ or closely matched modes for improved resolution and sensitivity under low pressure (high quality factor) ambients. However, perfect mode matching typically limits the bandwidth of the input rate to less than a few hertz which is unsuitable for many applications. An open-loop implementation with slightly mismatched modes trades off sensitivity for bandwidth. However, the scale factor is still inversely proportional to the difference between the drive and sense mode natural frequencies. These frequencies may be different functions of the ambient temperature and pressure, resulting in potential bias instability and scale factor drift. Closed-loop control has been cited as the solution to these issues by extending bandwidth without significantly degrading resolution. However, any control implementation is compounded by the severe challenge of resolving sub-angstrom displacements and maintaining system stability in the presence of larger perturbations (such as coupling of the driven motion of the proof mass) for a multi-degree of freedom system [3].

The current work utilizes resonant sensing as the detection principle for direct measurement of the Coriolis force. Resonant sensing involves the detection of an input measurand in terms of a resonant frequency shift in the sensing device. This mechanism of sensing has been widely used in a number of applications ranging from cantilevers in atomic force microscopes [4] to microaccelerometers [5]. Resonant sensing benefits from a direct frequency output, the ease of interfacing with digital signal processing, high resolution and large dynamic range. The specific application of resonant sensing to the gyroscope problem results in a number of additional benefits. The dynamics is considerably simplified from a minimally two degree of freedom system to a series of coupled single degree of freedom resonating elements. The scale factor turns out to be a dimensionless quantity that is dependent only on material and geometrical parameters. Resonant sensing has traditionally been hindered by sensitivity to environmental parameters such as temperature and pressure. However, the scale factor sensitivity to environmental variables is expected to be considerably lower for the resonant output gyroscope. 

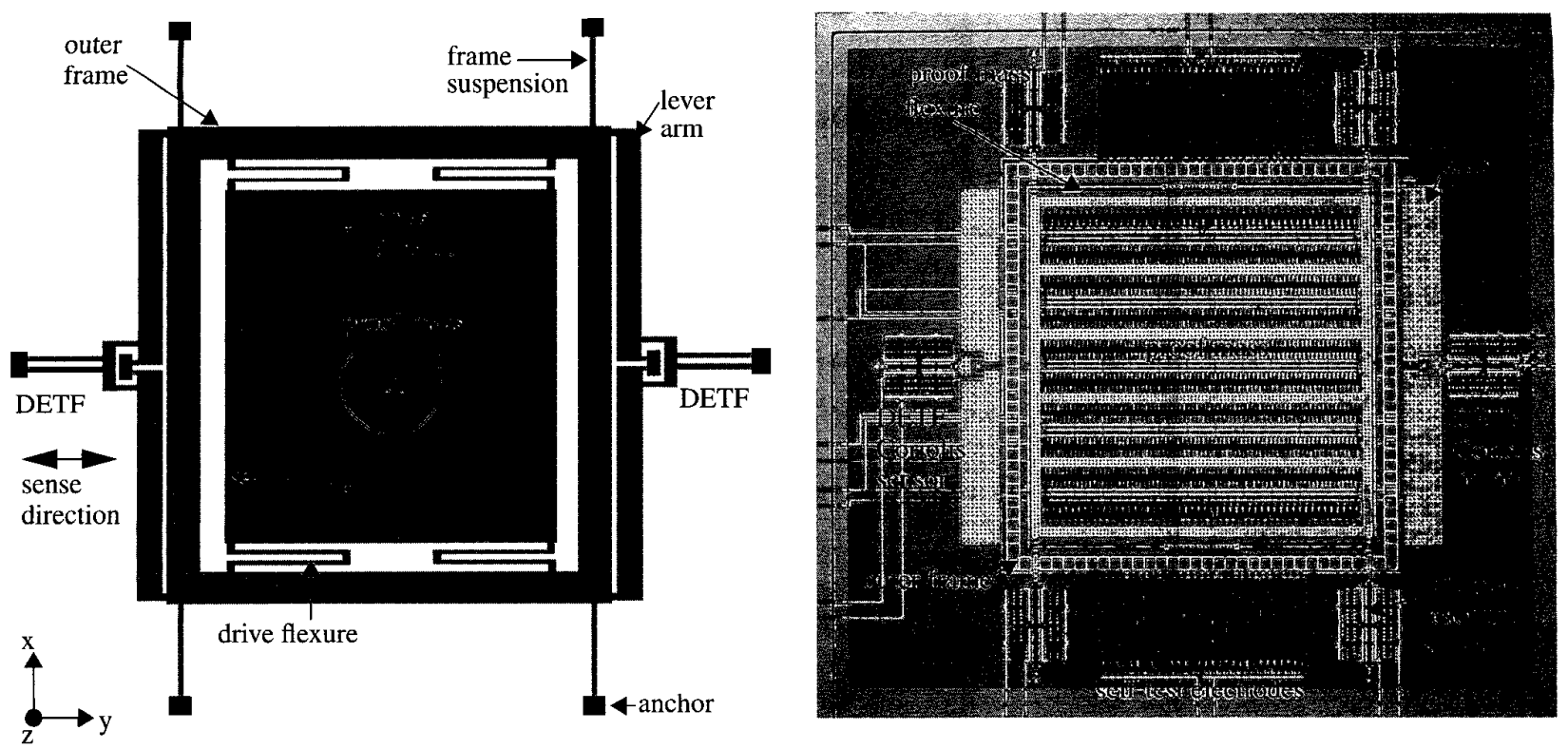

Figure 1: Pictorial depiction of the mechanical structure of the resonant output gyroscope. The figure on the left is a schematic of the mechanical structure illustrating the principle of operation and the figure on the right is a die photo of the device implementation in the Sandia National Laboratories IMEMS process.

\section{DEVICE DESCRIPTION}

A schematic of the $\mathrm{z}$-axis resonant output gyroscope is shown in Figure 1. The device consists of a proof mass suspended by flexures attached to a rigid frame. The proof mass is driven relative to the outer frame using embedded lateral comb drive actuators. Specialized combs can be employed for self-test and for quadrature error cancellation [2]. If an external rotation is applied to the chip about the $\mathrm{z}$ axis, the Coriolis force acting on the proof mass is transmitted to the outer frame. A lever mechanism[5] amplifies this force prior to being communicated axially onto two doubleended tuning fork (DETF) resonators placed on either side of the outer frame to provide for a differential output. The two tines of each tuning fork vibrate anti-phase to each other and parallel to the direction of motion of the inner proof mass. The periodic compression and tension of the tuning fork tines by the Coriolis force at the proof mass drive frequency modulates the resonant frequency of these force sensors. Each force sensor comprises of the tuning fork mechanical structure embedded in the feedback loop of an oscillator circuit. Thus, by demodulating the oscillator output frequency, the rotation rate applied to the device can be estimated.

\section{THEORY}

The dynamics of the device can be described by a series of coupled differential equations. The proof mass dynamics can be described for most part by a classical spring-massdamper equation. However, the dynamics of the DETF subjected to an axial time-varying Coriolis force is given by:

$$
\begin{gathered}
m_{r} \ddot{x}_{r}+b_{r} \dot{x}_{r}+\left(k_{r}+k_{1} \sin \left(\omega_{g} t\right)\right) x_{r}=F_{d} \\
k_{1}=c_{\text {mode }} \cdot \frac{A\left|F_{c}\right|}{2 L_{r}}
\end{gathered}
$$

We will use the subscripts, $r, g$ to designate parameter values for the DETF resonators and the gyroscope proof mass respectively. The significant perturbation term, $k_{1} \sin \left(\omega_{g} t\right)$, which represents a modulation of the spring constant of the resonant sensor at the gyroscope drive frequency $\left(\omega_{g} / 2 \pi\right)$ is directly contributed by the amplified Coriolis force impinging axially on the resonator tines. $F_{d}$ is the force that is applied to the tuning fork to sustain motion at resonance and essentially acts to cancel out the damping effects in the system. Thus, the Coriolis force $\left(F_{c}\right)$ modulates the spring constant of the resonator system. Equation (2) is also known as the Mathieu equation [6]. This equation has been widely studied in the context of parametric resonance, but in our case the proof mass drive frequency and the resonant frequency of the structure are designed so as to allow for stable operation only. An approximate solution of this equation is given by [6]:

$$
x_{r}=x_{o}\left(1+\beta \sin \left(\omega_{g} t\right)\right) \sin \left(\omega_{r} t-\beta \cos \left(\omega_{g} t\right)+\phi\right)
$$

Note that the displacement is both amplitude and frequency modulated. The modulation index, $\beta$, is representative of the Coriolis force and the approximation for narrow-band frequency modulation is valid as the magnitude of the stiffness variation for the applied Coriolis force is much smaller than the nominal stiffness i.e. $k_{1} \ll k_{r}$. The modulation index, $\beta$, can be expressed as the ratio of the peak frequency shift $(\Delta f)$ due to the applied force to the modulating Coriolis force frequency $\left(f_{g}\right)$ and is given by: 


$$
\beta=\frac{\Delta f}{f_{g}}=\frac{c_{\text {mode }} A F_{c}}{16 \pi^{2} f_{g} f_{r} L_{r} m_{r}}
$$

Note that the two tuning forks placed on either side of the structure experience an equal and opposite axial force. The output of the device is the resonant frequency shift difference ( $\delta f_{o}=\Delta f_{1}-\Delta f_{2}$ ) between the two tuning fork sensors, measured at the gyroscope proof mass drive frequency.

The scale factor of the sensor $(S)$ that relates the output frequency shift difference $\left(\delta f_{o}\right)$ between the two tuning forks to the external input rotation rate $\left(\Omega_{z}\right)$ is given by:

$$
S=\frac{\delta f_{o}}{\Omega_{z}}=\frac{c_{\text {mode }}}{2 \pi} \cdot A \cdot \frac{m_{g}}{m_{r}} \cdot \frac{f_{g}}{f_{r}} \cdot \frac{X_{g}}{L_{r}}
$$

Note that the scale factor is a dimensionless quantity relating an input rate to an output frequency shift difference. The expression is written in terms of a ratio of like quantities multiplied by a lever gain $(A)$ and a constant $\left(c_{\text {mode }}\right)$ dependent on the mode shape of the resonating element. The scale factor is only dependent on material and geometrical parameters and the displacement of the gyroscope proof mass $\left(X_{g}\right)$. The goal of the control scheme is now simplified to the requirement of maintaining constant amplitude motion for the gyroscope proof mass and the tuning fork force sensing elements at their respective resonant frequencies.

The other critical sensor parameter is the noise equivalent rate output of the sensor. This noise floor has electronic and mechanical constituents. We will consider oscillator electronic noise as the primary electronic noise $\left(\Omega_{n e}\right)$ component and ignore noise contributions from the signal processing scheme. The primary mechanical noise sources result from the brownian motion of the proof mass $\left(\Omega_{n p b}\right)$ and that of the tuning fork tines $\left(\Omega_{n r b}\right)$. An expression for the overall rotational rate equivalent noise density $\left(\Omega_{n}\right)$ can be written as:

$$
\overline{\Omega_{n}^{2}}=\overline{\Omega_{n p b}^{2}}+\overline{\Omega_{n p e}^{2}}+\overline{\Omega_{n r e}^{2}}+\overline{\Omega_{n r b}^{2}}
$$

\begin{tabular}{|l|c|}
\hline Noise source expression & $\begin{array}{c}\text { Design } \\
\text { value } \\
(\mathrm{deg} / \mathrm{sec} / \sqrt{H z})\end{array}$ \\
\hline $\bar{\Omega}_{n r b}=\sqrt{\frac{8 \pi^{2} m_{r} \omega_{r} k_{B} T}{\left(c_{m o d e} A m_{g} \nu_{g}\right)^{2} Q_{r}}\left(\frac{L_{r}}{X_{r}}\right)^{2}}$ & 0.04 \\
\hline $\bar{\Omega}_{n r e}=\frac{8 \pi \cdot m_{r} f_{r} L_{r}}{c_{m o d e} A m_{g} X_{g} \cdot S N R_{o s c}}$ & 0.2 \\
$\bar{\Omega}_{n p b}=\frac{\sqrt{4 k_{B} T b_{y}}}{\left(2 m_{g} \omega_{g} X_{g}\right)}$ & $<0.01$ \\
\hline $\bar{\Omega}_{n p e}=\sqrt{\left(\frac{V_{P}}{2 X_{g} m_{g} \omega_{g}} \frac{\partial C_{g}}{\partial y}\right)^{2} \bar{v}_{o n}^{2}}$ & 0.01 \\
\hline
\end{tabular}

Table 1: Contributions to noise equivalent rate output

The brownian noise of the resonating tuning fork tines is shaped by the resonator mechanical transfer function $\left(H_{r}\right)$. However, the electronic noise of the sustaining amplifier is not shaped by $H_{r}$ and sets the background noise of the oscillator away from the carrier frequency. Voltage noise on the electrodes that actuate the proof mass $\left(v_{o n}\right)$ can result in a noise force along the sensing direction for a non-ideal actuator. The noise contributions from the proof mass motion are low and typically the DETF oscillator noise sets the sensor resolution.

\section{DEVICE FEATURES}

The device dynamics has now been simplified to a series of coupled one-degree of freedom resonators. The requirement for multi-degree of freedom control disappear as the complexity is now transferred to the signal processing. The control goals are limited to sustaining motion in the inner proof mass and the DETF resonators at prescribed amplitudes. The scale factor of this device is defined as the ratio of the peak frequency shift difference between the two force sensors at the proof mass drive frequency to the rotation rate input (equation 5). This parameter is a dimensionless constant that is dependent only on geometrical design parameters and material constants. The mass and the frequency of the tuning forks and the proof mass are expected to have the same functional behavior, and hence the sensitivity of the scale factor to environmental variables due to these terms cancel out to first order. This assumption is strengthened by the fact that both the proof mass and tuning fork sensors oscillate along the same direction but it does require some degree of material uniformity across the structure. The scale factor sensitivity to an environmental variable, $x$, can now be written as:

$$
\frac{1}{S} \cdot \frac{\partial S}{\partial x}=\frac{1}{X_{g}} \frac{\partial X_{g}}{\partial x}-\frac{1}{L_{r}} \frac{\partial L_{r}}{\partial x}
$$

We are now left with a much smaller scale factor dependence in the resonator length $\left(L_{r}\right)$. For the specific case of $x$ representing temperature, this dependence is much smaller as compared to variations of parameters such as the Young's modulus and quality factor that are typically an order of magnitude or more higher. This variation can be potentially compensated by an amplitude gain control strategy applied to the gyroscope proof mass motion. To add to this benefit are the inherent advantages of resonant sensing, including a large dynamic range, high resolution and good linearity [2].

\section{EXPERIMENTAL CHARACTERIZATION}

A z-axis resonant output gyroscope was designed and fabricated in the Sandia National Laboratories integrated MEMS process [7]. A $2.25 \mu \mathrm{m}$ thick surface-micromachined polysilicon layer serves as the structural material and an underlying layer of polysilicon is used for electrical routing and shielding purposes. Sensor interface electronics are designed in a $5 \mathrm{~V} 2 \mu \mathrm{m}$ CMOS process. 


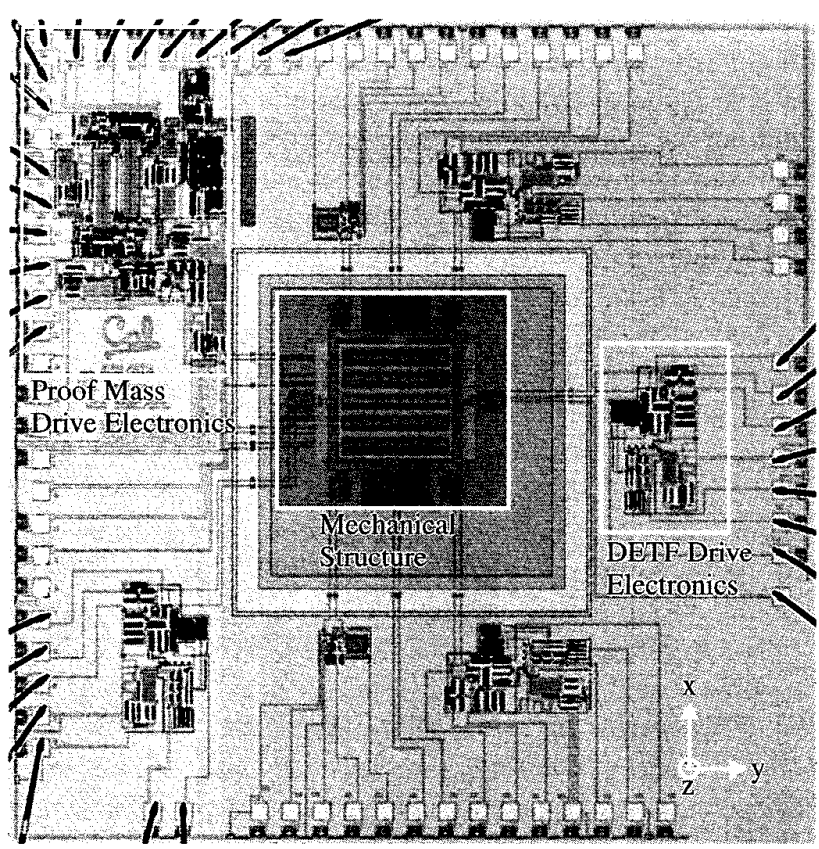

Figure 2: Die photo of the gyroscope module. The chip area is $4.5 \mathrm{~mm} \times 4.5 \mathrm{~mm}$.

A die photo of the gyroscope is shown in Figure 2. The device is comprised of an inner proof mass, an outer frame, a lever suspension, six double-ended tuning fork devices and an array of comb fingers for actuation, error compensation and other control and testing purposes. Two of the double-ended tuning forks are used for sensing the induced Coriolis force. The other four double-ended tuning forks, attached to the outer frame, are provided as references for temperature compensation and for drive motion measurements.

The designed scale factor of this device is $15 \mathrm{mHz} / \mathrm{deg} /$ $\mathrm{sec}$ and the estimated brownian noise equivalent rate is 0.04 $\mathrm{deg} / \mathrm{sec} / \sqrt{\mathrm{Hz}}$. On-chip circuits include a trans-resistance amplifier for driving the proof mass into oscillation and closed-loop Pierce oscillator circuits for the double-ended tuning fork resonators. The proof mass suspension was designed to be compliant to allow for large static deflection at operating voltages and a relatively low resonant frequency of $3.6 \mathrm{kHz}$. The double-ended tuning forks are designed for a resonant frequency of $300 \mathrm{kHz}$. The lever is designed to provide a force amplification factor of about 30 .

Substantial experimental characterization of the device has been completed. The observed magnitude response of the proof mass drive motion in air (Figure 3) was measured using the computer microvision system [8]. The observed resonant frequencies of the driven mode of the proof mass and the double-ended tuning forks lie slightly under designed values.

The Pierce oscillators exhibit a background noise floor of approximately $-100 \mathrm{dBc} / \mathrm{Hz}$ with in-circuit quality factors exceeding 30000 at $50 \mathrm{mtorr}$. When the proof mass as well as the two resonators are driven into motion, a component of the actuation force couples along the sense direction, resulting in a frequency modulated oscillator output as is shown in Figure 4. This coupling signal is in-phase with the Coriolis force and serves to introduce an offset in the output on the order of several hundred degrees per second. An off-chip phase-locked loop is used to demodulate the signal down to the drive frequency. Further conventional amplitude demodulation with the proof mass drive signal, followed by a lowpass filter directly results in a rotation rate voltage output. Figure 5 is a block diagram representation of the analog demodulation scheme currently implemented.

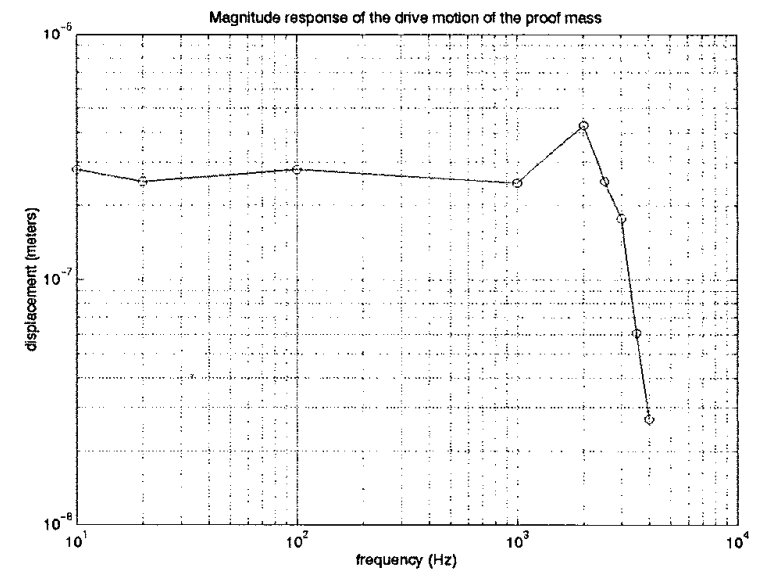

Figure 3: Magnitude response of the drive motion of the proof mass in air obtained using the computer microvision system [8]. The resonant frequency of the proof mass is $2.2 \mathrm{kHz}$ and the quality factor is about 5 at $1 \mathrm{~atm}$.

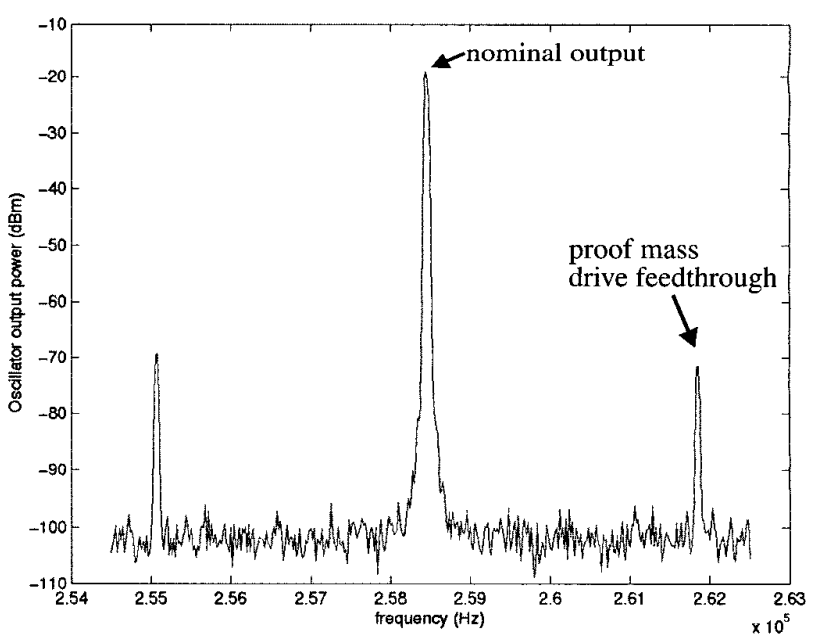

Figure 4: Output of the double-ended tuning fork device measuring the Coriolis force. The FM sidebands correspond to the coupling of the proof mass actuation force along the sense direction.

The device was tested on a rate table in a portable vacuum chamber that provided for ambient pressures between 10-250 mtorr. However, due to the requirement of a vacuum feedthrough, the device could not be subjected to a fixed constant rotation rate. When an external sinusoidal rotation 
rate is applied to the device, the oscillator sideband output is modulated by the Coriolis force as shown in Figure 6. Several prototypes that were tested over a range of rotation rates between 0 and $25 \mathrm{deg} / \mathrm{sec}$. at frequencies of between $5 \mathrm{~Hz}$ and $15 \mathrm{~Hz}$ exhibited noise floors of approximately $0.3 \mathrm{deg} /$ $\mathrm{sec} / \sqrt{\mathrm{Hz}}$, limited by electronic noise of the oscillator circuit. Scale factor characterization of the device is underway.

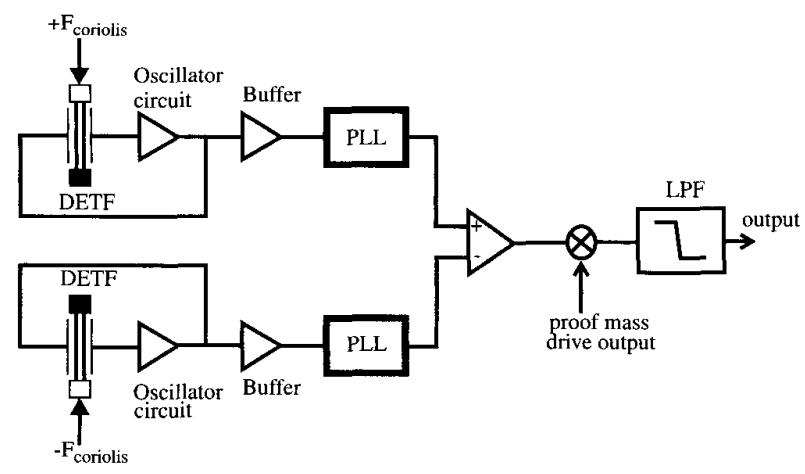

Figure 5: Block diagram of the signal processing electronics used to extract the rotation rate output signal from the DETF oscillator output.

\section{SUMMARY}

An implementation of a microelectromechanical resonant output gyroscope has been described. The resulting benefits include simpler dynamics and control, improved scale factor stability over micromechanical gyroscopes utilizing open-loop displacement sensing, large dynamic range and high resolution.

A device was designed and fabricated in the Sandia National Laboratories IMEMS process. The design emphasis was on provision for investigation of dynamics and proof-of-concept demonstration. Considerable room for design optimization remains and an improved and optimized second generation device is currently being fabricated at the Analog Devices MEMS fabrication facility.
A prototype device, fabricated at Sandia National Laboratories, exhibited a noise floor of $0.3 \mathrm{deg} / \mathrm{sec} / \sqrt{\mathrm{Hz}}$.

\section{ACKNOWLEDGEMENTS}

The work was partially supported by DARPA grants F30602-97-2-0266 and F30602-97-C-0127. The devices were fabricated at Sandia National Laboratories. Thanks go to Joe Silva at Melgar Photographers for the die photos.

\section{REFERENCES}

[1] N. Yazdi et al., Micromachined Inertial Sensors, Proc. IEEE, August 1998, pp. 1640-1659.

[2] W. Clark et al., Surface micromachined Z-axis vibratory rate gyroscope, Proc. Solid State Sensors and Actuators Workshop, 1996, pp. 283-7.

[3] A. Shkel et al., Dynamics and control of micromachined gyroscopes, Proc. American Control Conference, 1999, pp. 2119-24.

[4] T. Albrecht et al., Frequency modulation detection using high-Q cantilevers for enhanced force microscope sensitivity, J. Appl. Physics, 69(2), 15 Jan. 1991, pp. 668-673.

[5] T. Roessig et al., Surface-micromachined resonant accelerometer, Ninth International Conference in Solid State Sensors and Actuators, 1997, pp. 859-862.

[6] N. McLachlan, Theory and Application of Mathieu Functions, Clarendon Press, 1947.

[7] J. Smith et al., Embedded micromechanical devices for the monolithic integration of MEMS with CMOS, IEDM Tech. Digest, 1995, pp. 609-612.

[8] D. Freeman et al., Multidimensional motion analysis of MEMS using computer microvision, Proc. Solid State Sensors and Actuators Workshop, 1998, pp. 150-155.
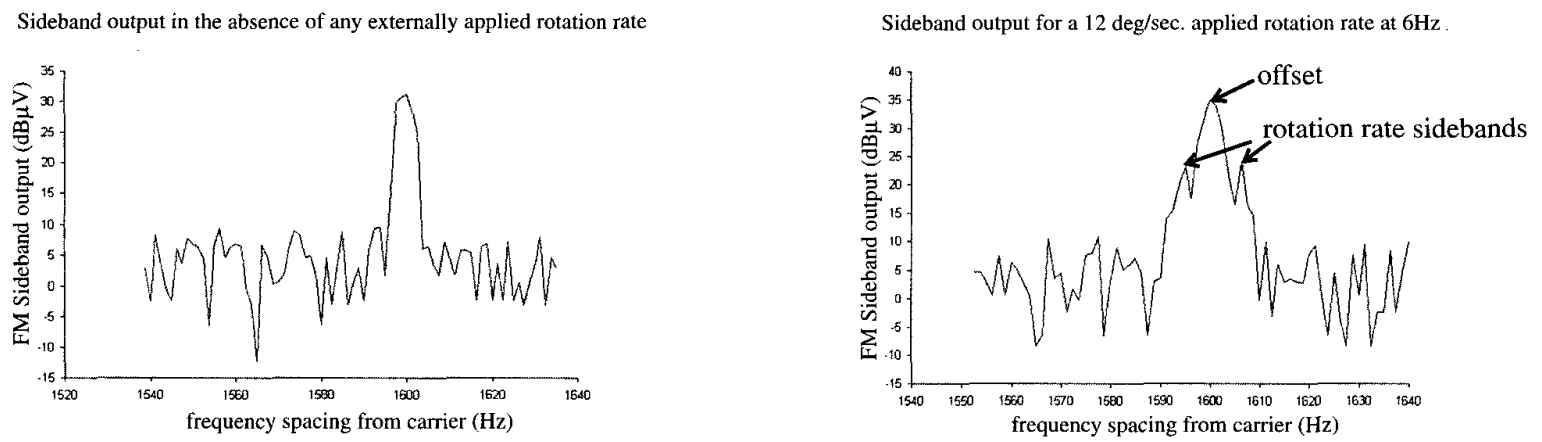

Figure 6: The frequency scale in the graphs shown above has been zoomed in on one of the FM sidebands of the oscillator output depicted in Figure 4. In the absence of an externally applied rotation rate, the sideband output solely reflects the coupling of the actuation force along the sense direction. In the presence of an applied sinusoidal rotation rate (12 deg/sec. at $6 \mathrm{~Hz})$, the sideband output is amplitude modulated by the Coriolis signal as shown. The proof mass is driven at $1600 \mathrm{~Hz}$. 